\title{
Transport injuries and deaths in the Eastern Mediterranean Region: findings from the Global Burden of Disease 2015 Study
}

\author{
GBD 2015 Eastern Mediterranean Region Transportation Injuries Collaborators ${ }^{1}$
}

Received: 2 May 2017/Revised: 23 May 2017/ Accepted: 29 May 2017/Published online: 3 August 2017

(C) The Author(s) 2017. This article is an open access publication

\begin{abstract}
Objectives Transport injuries (TI) are ranked as one of the leading causes of death, disability, and property loss worldwide. This paper provides an overview of the burden of TI in the Eastern Mediterranean Region (EMR) by age and sex from 1990 to 2015.

Methods Transport injuries mortality in the EMR was estimated using the Global Burden of Disease mortality database, with corrections for ill-defined causes of death, using the cause of death ensemble modeling tool. Morbidity estimation was based on inpatient and outpatient datasets, 26 cause-of-injury and 47 nature-of-injury categories.

Results In 2015, 152,855 (95\% uncertainty interval: 137,900-168,100) people died from TI in the EMR countries. Between 1990 and 2015, the years of life lost (YLL) rate per 100,000 due to TI decreased by $15.5 \%$, while the years lived with disability (YLD) rate decreased by $10 \%$,
\end{abstract}

This article is part of the supplement "The state of health in the Eastern Mediterranean Region, 1990-2015”.

The members of GBD (Global Burden of Disease) 2015 Eastern Mediterranean Region Transportation Injuries Collaborators are listed at the end of the article. Ali H. Mokdad, on behalf of GBD 2015 Eastern Mediterranean Region Transportation Injuries Collaborators, is the corresponding author.

Electronic supplementary material The online version of this article (doi:10.1007/s00038-017-0987-0) contains supplementary material, which is available to authorized users.

GBD 2015 Eastern Mediterranean Region Transportation Injuries Collaborators

mokdaa@uw.edu

1 Institute for Health Metrics and Evaluation, 2301 5th Avenue, Suite 600, Seattle, WA 98121, USA and the age-standardized disability-adjusted life years (DALYs) rate decreased by $16 \%$.

Conclusions Although the burden of TI mortality and morbidity decreased over the last two decades, there is still a considerable burden that needs to be addressed by increasing awareness, enforcing laws, and improving road conditions.

Keywords Transport injuries · Eastern Mediterranean Region - Burden of disease

\section{Introduction}

Transport injuries (TI) are a major cause of global mortality and morbidity. In 2015, they caused 1.5 million deaths globally [95\% Uncertainty Interval (UI) 1.4-1.5 million] (Wang et al. 2016). In addition to deaths on the roads, up to 50 million people incur nonfatal injuries each year as a result of road traffic crashes and other accidents (GBD 2015 Disease and Injury Incidence and Prevalence Collaborators 2016). The significance of this public health threat is most pronounced in low- and middle-income countries (LMIC), where $90 \%$ of the world's road trafficrelated deaths take place. It is projected to be the fifth leading cause of mortality around the world through the year 2030 (Naeem 2010). Transport injuries also exert a significant impact on the affected families, health care services, and national economies (Ainy et al. 2014). Moreover, TI are estimated to cause approximately 3\% loss of gross domestic product (GDP) in LMIC (WHO 2015).

The causes of TI can be attributed to different factors: excessive speed, consumption of drugs and alcohol, failure to enforce the use of protective measures such as seatbelts and helmets, poor vehicle impact protection, and poor road conditions (Keay and Simmonds 2005). The road user, the 
vehicle, and the built environment are elements of a dynamic system that work together to either produce or prevent injuries. Many factors can also influence the frequency and nature of road crashes, including weather conditions, school holidays, time of the day, and alcohol consumption (Sukhai et al. 2011; Karacasu et al. 2011).

The Eastern Mediterranean Region (EMR) contains over 600 million people and consists of 22 countries with varying levels of national income: Afghanistan, Arab Republic of Egypt, Bahrain, Djibouti, Iraq, Islamic Republic of Iran, Jordan, Kingdom of Saudi Arabia (KSA), Kuwait, Lebanon, Libya, Morocco, Oman, Pakistan, Palestine, Qatar, Republic of Yemen, Somalia, Sudan, Syrian Arab Republic (Syria), Tunisia, and the United Arab Emirates (UAE). Although the overall number of registered vehicles per 1000 population is comparatively low (96 per 1000 population), the case-fatality rate from TI is one of the highest in the world (WHO Regional Office for the Eastern Mediterranean 2017). Despite this, studies surrounding this topic are scarce, and reliable data are limited. In addition to their fatality burden, road traffic crashes also increase the burden of nonfatal injuries (Chandran et al. 2010). According to the World Health Organization (WHO) estimates, TI were ranked as the sixth leading cause of death in the EMR, surpassing tuberculosis, malaria, and HIV/AIDS, and the region has the second highest road traffic fatality rate in the world (Kassebaum et al. 2016). In this manuscript, we assessed the burden of TI in the EMR by age and sex from 1990 to 2015, and compared the burden to the global TI, from the Global Burden of Diseases, Injuries, and Risk Factors Study 2015.

\section{Methods}

Transport injuries estimates included pedestrian, cyclist, motorcyclist, and motor vehicle road injuries, in addition to other water and air transport injuries. GBD 2015 estimated injury mortality from vital registration, verbal autopsy, mortality surveillance, censuses, surveys, and police record data. Police and crime reports were used as data sources only for the estimation of deaths from road traffic injuries (Wang et al. 2016). The police data were collected from published studies, national agencies, and institutional surveys such as the United Nations Crime Trends Survey and the WHO Global Status Report on Road Safety Survey. For countries with vital registration data we did not use police records, except if the recorded number of road injury deaths from police records exceeded that in the vital registration.

We assessed mortality by mapping all data sources to the GBD cause list of diseases and injuries, and then adjustments were made for ill-defined causes of death, or garbage codes. Finally, ensemble models with varying choices of covariates and mathematical models were run using the GBD Cause of Death Ensemble modeling (CODEm) software to derive estimates by age, sex, country, year, and cause. Final fatal discontinuity estimations for these causes were merged with CODEm results postCause of Death Correct (CoD Correct) to produce final cause of death results. CoD Correct is a process that uses a simple algorithm to scale all cause-specific deaths from all causes for each age group, sex, year, and location, and thereby ensures that the sum equals total all-cause mortality (Wang et al. 2016).

The preparation of cause of death data, the redistribution of garbage codes, the modeling process, and covariates are explained in more detail elsewhere (Wang et al. 2016). The International Classification of Diseases (ICD) was used to classify injuries. In GBD 2015, injury incidence and deaths are defined as ICD-9 codes E000-E999 and ICD-10 chapters V-Y. More details can be found in a full description of GBD 2015 study methodology (Wang et al. 2016).

We estimated incidence of injury warranting inpatient admission ("inpatient care") and incidence of injury warranting other types of care ("outpatient care") for all causeof-injury categories. Injuries warranting inpatient care refer to injury cases of sufficient severity to require inpatient care assuming no restrictions in access to health care. More details about data sources and our strategy to assess the nonfatal burden of disease can be found elsewhere (Kassebaum et al. 2016).

We calculated years of life lost (YLLs) by multiplying deaths by the residual expected individual life span at the age of death as derived from the GBD 2015 standard model life table (Wang et al. 2016). Years lived with disability (YLDs) were calculated by multiplying the number of prevalent cases of a certain health outcome by the disability weight assigned to this health outcome. A disability weight reflects the magnitude of the health loss associated with an outcome and has a value that is anchored between 0 , equivalent to full health, and 1 , equivalent to death. Disability-adjusted life years (DALYs) were calculated by adding YLLs and YLDs.

We evaluated the associations between TI and sociodemographic status using the Socio-demographic Index (SDI). SDI is a composite measure developed for GBD 2015 that accounts for fertility rate, lag-dependent income per capita, and education (Wang et al. 2016). To capture the average relationships for each age-sex group, we applied a simple least squares spline regression of the TI mortality rate on SDI. The SDI is scaled from 0 to 1 , where 0 represents the lowest possible observed SDI and 1 is the highest. We reported uncertainty for all our estimates (Kassebaum et al. 2016), which have varying degrees of uncertainty arising from input data, the data adjustments and the statistical models. We have propagated uncertainty 
from all these sources using standard GBD methods of repeating all calculations 1000 times, each time drawing from distributions rather than point estimates for all the relevant parameters in our models (Kassebaum et al. 2016). For the injury mortality estimates the estimation of model uncertainty is inherent to the ensemble modeling method (Wang et al. 2016).

\section{Results}

\section{Mortality}

In 2015, there were 152,855 deaths due to TI in EMR (UI 137,873-168,097), and 1,466,557 deaths globally (UI $1,394,757-1,536,454)$. In 2015 , TI was the eighth leading cause of death in EMR, but the second leading cause of death in Qatar, Oman, and UAE. The percentage of deaths of TI out of total deaths was the highest in Qatar (20\%), Oman (16\%), and UAE (14\%), and the lowest in Pakistan $(1.9 \%)$, Lebanon $(1.8 \%)$, and Somalia (1.5\%). TI accounted for 27.8 (UI 25.1-30.5) deaths per 100,000 population, higher than the global mortality rate of 20.24 (UI 9.3-21.2) per 100,000 population (Wang et al. 2016). For the region, TI accounted for $3 \%$ of all deaths.

In 2015, Afghanistan had the highest mortality rate in the region at 66.2 (UI 48.7-87.9) and Lebanon had the lowest at 8.5 (UI 5.7-12.7) per 100,000 population (Fig. 1; Table 1).
From 1990 to 2015 Libya, Pakistan, and Egypt had 37, 14.8, and $12.4 \%$ increases in TI mortality rate, respectively (Table 1). Three countries, UAE, Kuwait, and Qatar, have a high SDI score, with TI mortality rates significantly higher in UAE (36.8 per 100,000) and Qatar $(29.99$ per 100,000$)$ than the global average for high-income countries (12.2 per 100,000). Motor vehicle road injuries and pedestrian road injuries were the leading causes for age-standardized mortality rates in the region in 2015 (Fig. 2).

Males were substantially more affected than females, with an overall mortality rate of 43.6 (UI 38.5-48.5) per 100,000 , compared to a rate of 11.36 (UI 10.1-12.9) per 100,000 for females (Fig. 2). The ratio of age-standardized TI mortality between males and females in the EMR was 3.84 compared to 3.1 globally. Within the EMR, Pakistan had, by far, the highest ratio of mortality in males to females at 12.0, with the next being Kuwait at 4.5.

Table 2 shows observed-to-expected (based exclusively on SDI) ratios for sub-causes of TI by country in 2015. The observed-to-expected ratio varied substantially across both country and sub-cause. The rate of pedestrian injuries in Oman was nearly four times higher than expected. Pedestrian injuries in Oman had a ratio of 4.9, an observed 37.9 (UI 30.7-46.3) deaths per 100,000 compared to an expected 7.66. Only in Oman were pedestrian injuries the largest sub-cause of TI (Fig. 2). Afghanistan, Iran, and Qatar all had observed values for multiple sub-causes that greatly exceeded the expected values (Table 2 ).

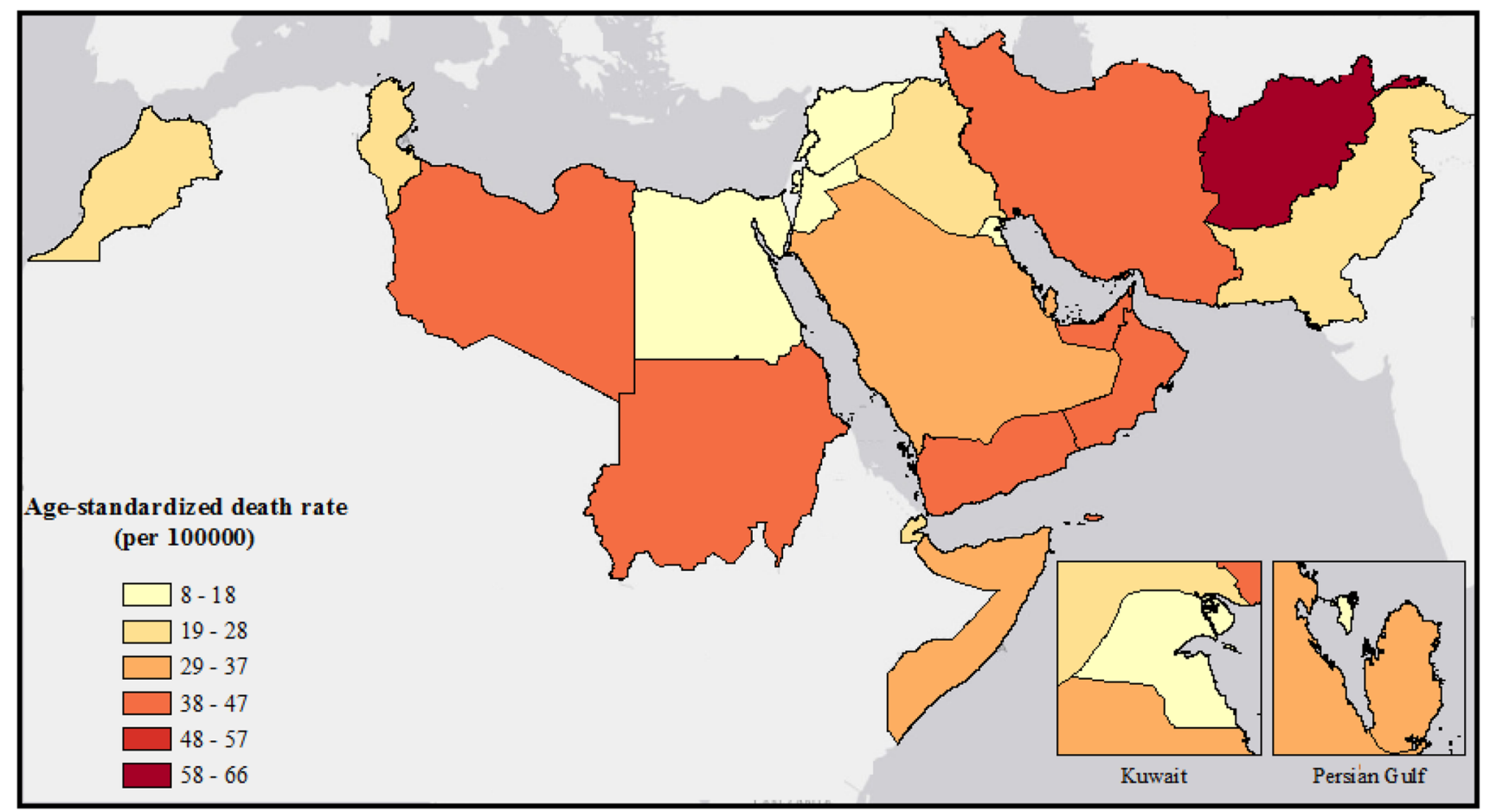

Fig. 1 Map of age-standardized mortality rates for transport injuries in the Eastern Mediterranean Region, both sexes, in 2015. (Global Burden of Disease Study 2015, Eastern Mediterranean Countries, 2015) 


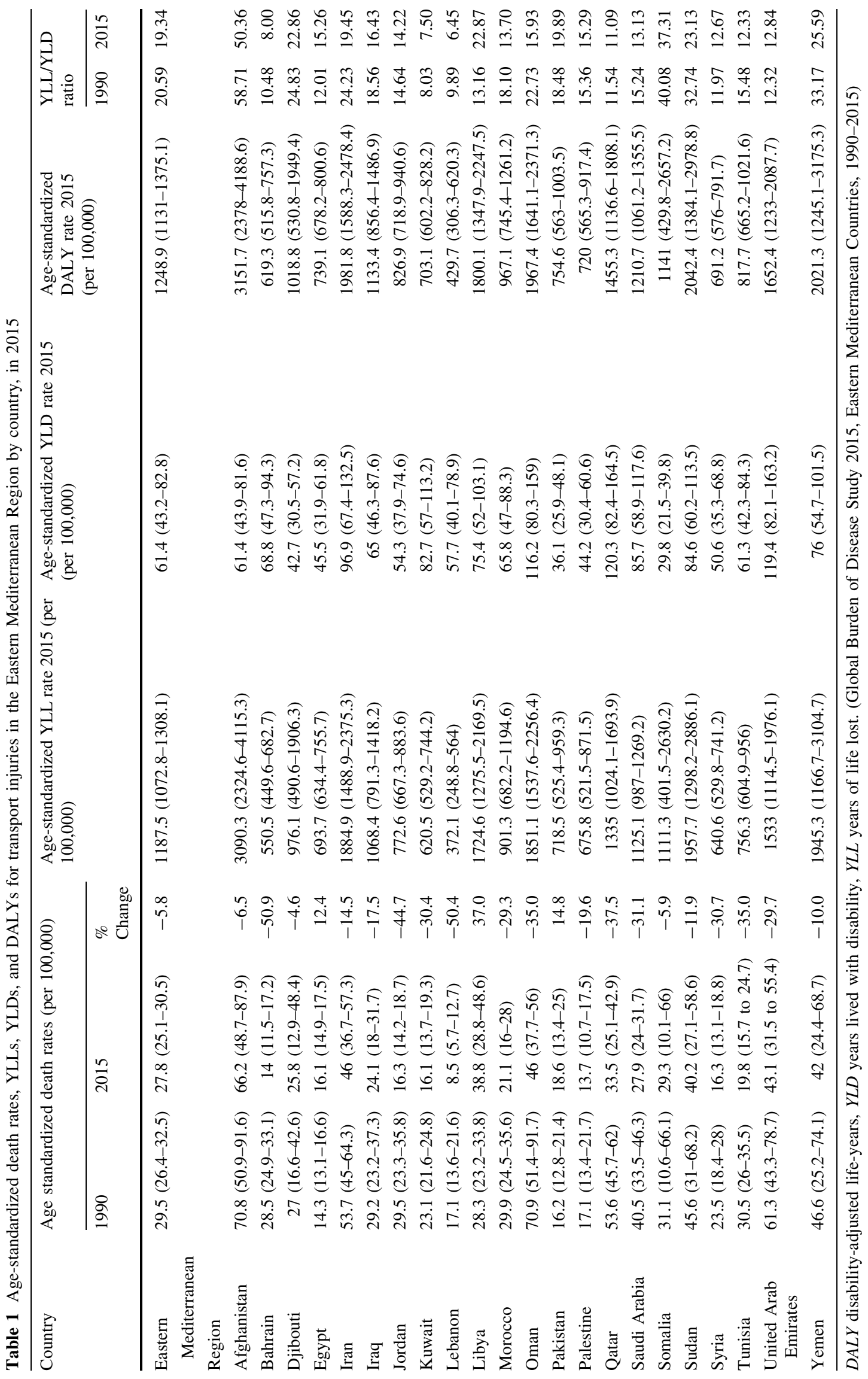




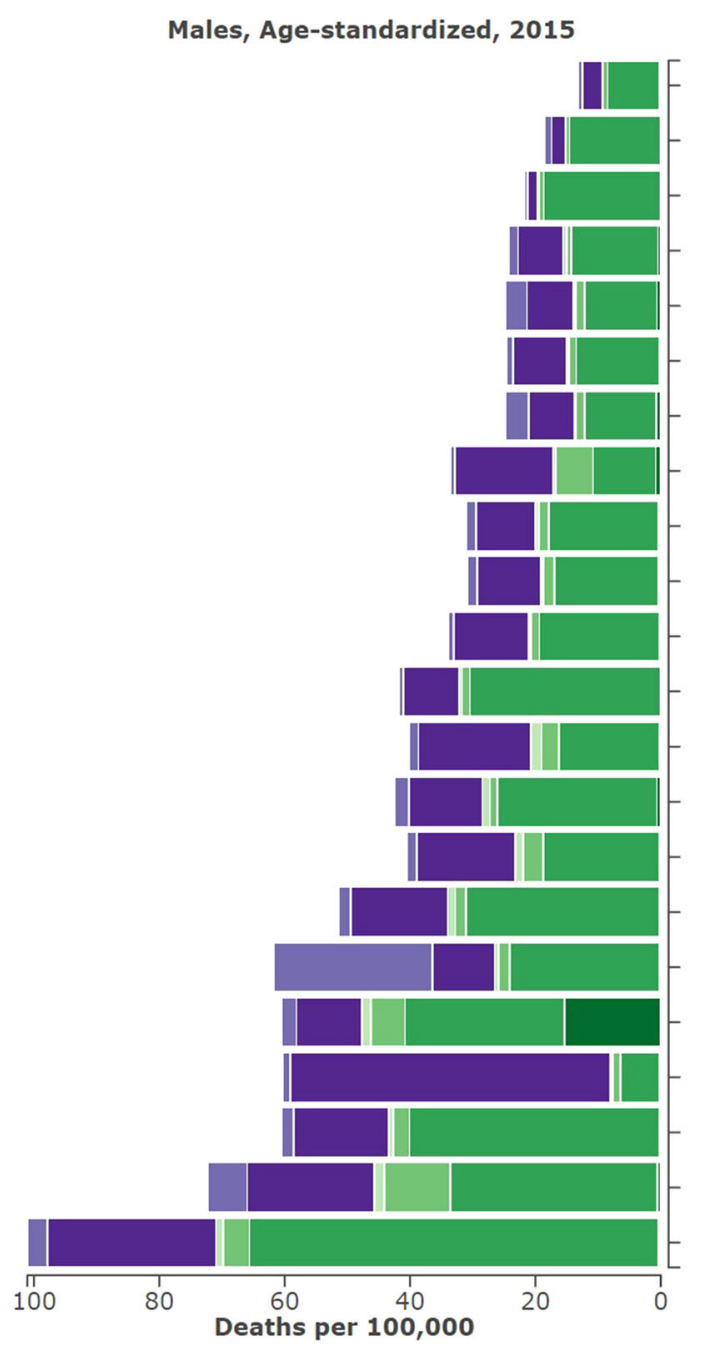

Females, Age-standardized, 2015

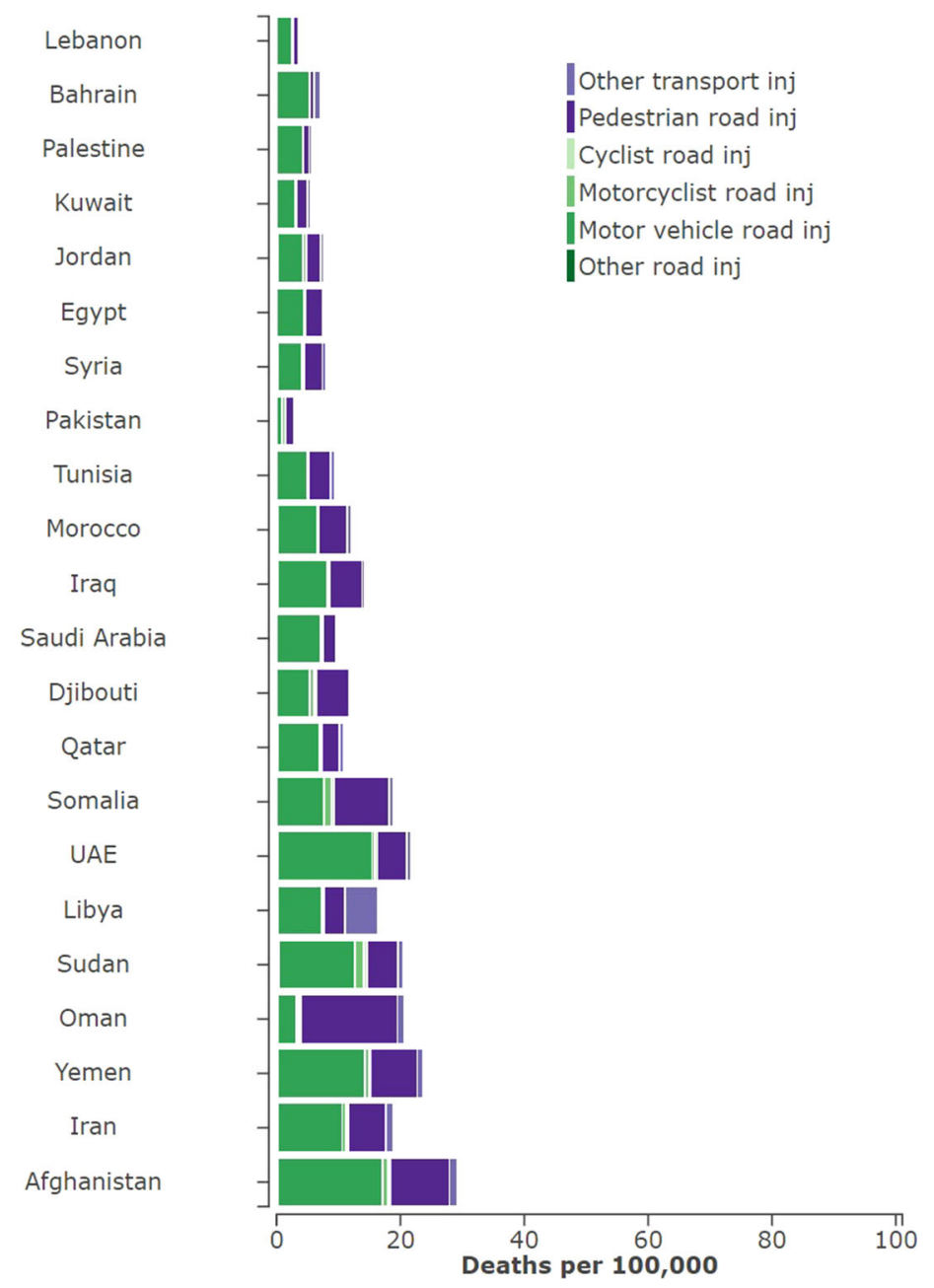

Fig. 2 Age-standardized mortality rates for sub-causes of transport injuries in the Eastern Mediterranean Region, by sex and by country, in 2015. (Global Burden of Disease Study 2015, Eastern Mediterranean Countries, 2015)

\section{Years of life lost (YLLs)}

The rate of age-adjusted YLLs per 100,000 population was significantly higher in the EMR than globally, 1187.5 (UI 1072.8-1308.1) compared to 881.2 (UI 837.6-923.2) per 100,000 population (Table 1). By age, YLLs peaked in the 20-24 age group, and then steadily decreased as age increased (Fig. 3). This was consistent with the global trend. By country, Afghanistan had the highest age-standardized YLL rate at 3090.3 (UI 2324.6-4115.3) per 100,000 population, nearly three times the regional average (Table 1).

\section{Years lived with disability (YLDs)}

Unlike YLLs, the rate of age-adjusted YLDs in the EMR was lower than the global average, 61.4 (UI 43.2-82.8) compared to 90.2 (UI 63.0-122.2) (Wang et al. 2016). The percentage of YLDs attributable to TI was also lower in the
EMR than globally. YLDs steadily increased with age (Fig. 3). Oman, Qatar, and the UAE had the highest agestandardized YLD rates at 116.2, 120.3, and 119.4, respectively (Table 1). Somalia had the lowest rate, at 29.8 (UI 21.5-39.8) (Table 1). In all countries, motor vehicle injuries were the leading sub-cause of YLDs (Table 1).

The YLL/YLD ratio in the EMR for 2015 was 19.34, almost double the global ratio of 9.77 (Wang et al. 2016). By country, Afghanistan had the highest ratio at 50.36, and only Kuwait and Lebanon were below the global ratio at 7.50 and 6.45 , respectively (Table 1). For all age groups and countries, YLLs were the primary contributor to DALYs in terms of TI.

\section{Disability-adjusted life years (DALYs)}

In 2015, TI were the eleventh leading cause DALYs, causing 8,069,712 (95\% UI 7,303,759-8,888,094) DALYs. 
Table 2 Ratio of observed mortality rates to expected mortality rates on the basis of SDI alone for sub-causes of transport injuries in the Eastern Mediterranean Region, by country, in 2015. (Global Burden of Disease Study 2015, Eastern Mediterranean Countries, 2015)

\begin{tabular}{|c|c|c|c|c|c|c|}
\hline \multirow[t]{2}{*}{ Country } & \multicolumn{6}{|c|}{ Observed/expected age-standardized death rates for transport injuries 2015} \\
\hline & Pedestrian & Cyclist & Motorcyclist & Motor vehicle & Other road injuries & Other transport injuries \\
\hline Afghanistan & 1.319 & 1.005 & 0.918 & 3.511 & 2.151 & 1.678 \\
\hline Bahrain & 0.265 & 0.095 & 0.150 & 1.156 & 0.682 & 0.985 \\
\hline Djibouti & 0.683 & 1.192 & 0.499 & 0.878 & 0.744 & 0.561 \\
\hline Egypt & 0.403 & 0.244 & 0.172 & 0.721 & 0.498 & 0.501 \\
\hline Iran & 1.693 & 0.975 & 2.088 & 2.202 & 1.948 & 3.107 \\
\hline Iraq & 0.524 & 0.264 & 0.210 & 1.073 & 0.687 & 0.471 \\
\hline Jordan & 0.555 & 0.195 & 0.319 & 0.751 & 0.611 & 1.567 \\
\hline Kuwait & 1.995 & 0.562 & 0.263 & 2.115 & 1.651 & 1.402 \\
\hline Lebanon & 0.324 & 0.126 & 0.168 & 0.610 & 0.433 & 0.465 \\
\hline Libya & 0.561 & 0.360 & 0.318 & 1.340 & 0.849 & 10.873 \\
\hline Morocco & 0.436 & 0.237 & 0.278 & 0.931 & 0.598 & 0.683 \\
\hline Oman & 4.955 & 0.321 & 0.295 & 0.483 & 1.938 & 0.897 \\
\hline Pakistan & 0.502 & 0.294 & 0.929 & 0.453 & 0.533 & 0.260 \\
\hline Palestine & 0.077 & 0.154 & 0.118 & 0.892 & 0.387 & 0.286 \\
\hline Qatar & 1.905 & 0.828 & 0.333 & 2.402 & 1.851 & 1.539 \\
\hline Saudi Arabia & 0.937 & 0.309 & 0.319 & 2.228 & 1.434 & 0.510 \\
\hline Somalia & 1.040 & 1.245 & 0.891 & 1.060 & 1.036 & 1.013 \\
\hline Sudan & 0.467 & 1.129 & 1.036 & 1.646 & 1.197 & 0.971 \\
\hline Syria & 0.309 & 0.202 & 0.214 & 0.604 & 0.414 & 1.342 \\
\hline Tunisia & 0.584 & 0.331 & 0.285 & 0.977 & 0.707 & 0.765 \\
\hline United Arab Emirates & 5.292 & 1.399 & 0.725 & 5.881 & 4.362 & 1.759 \\
\hline Yemen & 0.705 & 0.543 & 0.505 & 2.373 & 1.278 & 0.911 \\
\hline
\end{tabular}

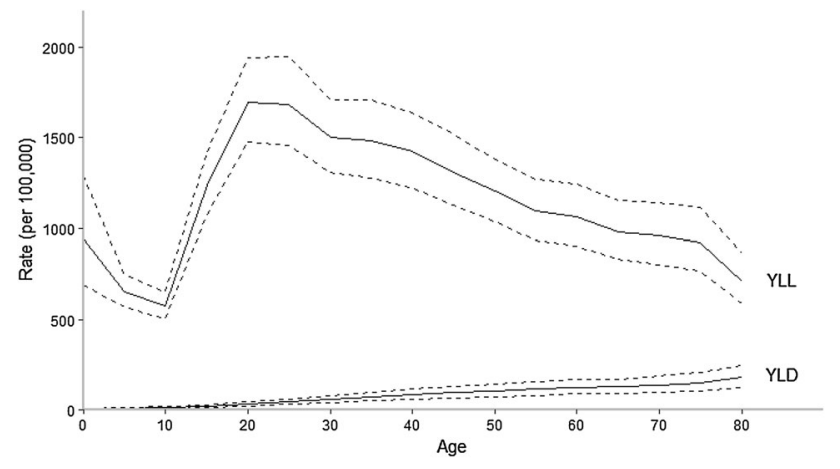

Fig. 3 YLL and YLD rates for transport injuries in the Eastern Mediterranean Region, both sexes, in 2015. YLD years lived with disability, YLL years of life lost. (Global Burden of Disease Study 2015, Eastern Mediterranean Countries, 2015)

The highest and lowest age-standardized rates of DALYs were observed in Afghanistan and Sudan. The TI DALY age-standardized rates in 2015 , were higher $(3.1 \%$ of all DALYs) compared to $0.3 \%$ of the total number of DALYs from disease globally (Wang et al. 2016). Overall, there was a $9.9 \%$ increase from 1990 to 2015 for DALYs attributable to TI in EMR. From 1990 to 2015 DALY rates decreased in all countries except Pakistan and Libya, which increased $6.64 \%$ and $23.9 \%$, respectively (Table 1; (Fig. 4). For all age groups and countries, YLLs were the primary contributor to DALYs (Fig. 4).

\section{Discussion}

Our study is the first to report on the burden of TI in the EMR from 1990 to 2015. Our results show that EMR mortality rates due to TI have not fallen as quickly as the global estimates. Three countries-Libya, Pakistan, and Egypt - even have had increases in death rates. Our results show that $\mathrm{TI}$ are still a major health problem in the region and call for serious efforts to reduce their burden.

The YLL to YLD ratio can be used as an indicator for the severity of TI and the effectiveness of health system intervention. The higher this ratio is, the more severe and fatal the crashes are and the less effective interventions the health system provides are. This ratio may suggest that health care access and interventions are not up to global standards in the region, in spite of the economic growth 


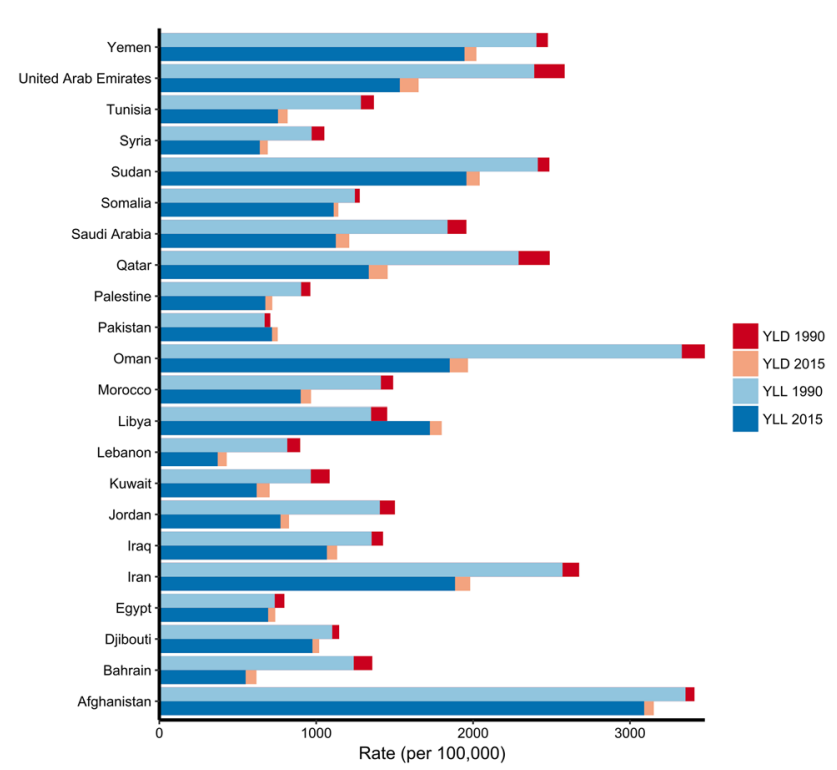

Fig. 4 DALY rates per 100,000 population for transport injuries in the Eastern Mediterranean Region both sexes combined, by country, in 1990 and 2015. DALY disability-adjusted life-years, YLD years lived with disability, $Y L L$ years of life lost. (Global Burden of Disease Study 2015, Eastern Mediterranean Countries, 1990-2015)

and high SDI of some countries in the EMR. It has been shown that improved access to better-quality trauma care systems has played a role in the decreasing mortality rates due to TI in high-income countries (Noland 2003).

Most of the research on the impact of trauma care comes from high-income countries where systems have been implemented with few resource constraints. Assessments in LMICs have consistently identified enormous gaps in the resources needed to provide adequate care for the injured (Reynolds et al. 2017). A review describing reports that evaluated the impact of trauma care systems and system components in LMICs, identified reports from 32 countries. These reports, which describe potentially useful interventions to strengthen care for the injured in LMICs, were found in only about one-quarter of LMICs. The study suggests a substantial research gap that spans all regions (Reynolds et al. 2017). Another study suggested that mortality among people with life-threatening but potentially survivable injuries was sixfold lower in high-income countries $(6 \%)$ than in low-income countries $(36 \%)$ (Mock et al. 1993).

Globally, the burden of disease due to TI has decreased significantly since 1990 , but this decrease is largely in high-income regions, with the reverse trend occurring in low-income and middle-income countries. Some studies have suggested that this is due to the growth in motorization and traffic density outpacing infrastructural development and levels of law enforcement (Ameratunga et al. 2006; Naghavi et al. 2009; WHO 2013). Countries with fast-growing economies have experienced rapid economic development that led to changes in lifestyle and environment and subsequently impacted health and mortality (Razzak et al. 2004; Luoma and Sivak 2012; Hyder and Vecino-Ortiz 2014). Motorization is rapidly increasing in the region (WHO 2015), and our study suggests that many regulations should be implemented. Safer roads, enforced traffic laws, formal driver education with more stringent driver license procedures and policies, and safe vehicle regulations need to be rigorously implemented to cope with the increase in access to vehicles, especially in high-income countries in the region. Countries with high numbers for specific causes, like pedestrian injuries in Oman, should implement specific measures to protect those at risk.

Similar to global trends (GBD 2015 Disease and Injury Incidence and Prevalence Collaborators 2016; Kassebaum et al. 2016; Wang et al. 2016), TI in the EMR disproportionately affect individuals who are in the economically productive age group of 15-44. This exerts an added pressure on the national economies of the EMR countries, especially those with limited resources (Mokdad et al. 2014, 2016 ). The burden of TI is significantly higher in males than females. This gender ratio is consistent with global trends (Wang et al. 2016). Besides being a public health burden, TI are also associated with an immense economic burden; it is estimated to cost EMR countries a total of US\$7.5 billion per year, equivalent to $1-1.5 \%$ of the GDP of most countries in the region (Bishai et al. 2006).

Despite the continuous threat of the burden of injuries in the EMR, few studies have been conducted to assess the burden of TI in the region, with the available ones being limited to small-scale, city-based, or facility-based studies. Coverage of vital registration is low or absent in large parts of the EMR and issues of incompleteness and differences in death certification systems, definitions of variables, and methods of data collection usually compromise the quality of data (Setel et al. 2007; Mahapatra et al. 2007; Obermeyer et al. 2010; Joubert et al. 2012). In our study, it was necessary to predict estimates using models, relying on covariates and verbal autopsy data (Noland 2003; Kassebaum et al. 2016). We added police and mortuary data for TI to help predict level and age patterns in countries with sparse or absent cause of death data, even though we know from countries with nearcomplete vital registration data that police records tends to underestimate the true level of deaths. The large GBD mortality database allows us to use statistical models that can borrow strength when data is missing from similar countries, previous years, published literature if no raw data is available, published reports, police reports, media, etc. Although this ensures an estimate for all causes and all countries, estimates for populations and time periods with sparse or absent data are inherently less precise. 
While we attempt to capture all sources of uncertainty from sampling error, non-sampling error, and model specifications in the $95 \%$ uncertainty intervals, additional sources of uncertainty may not have been captured (Mathers et al. 2006; Byass et al. 2013).

A study in Saudi Arabia showed that rates of death from road traffic accidents based on police reports and on health registration data are different, and that unlike police-reported data, health registration does not show steadiness or decline in the rates of road traffic deaths (Barrimah et al. 2012). These inconsistencies may be caused by differences in definitions, or may reflect differences in data collection methods (Loo and Tsui 2007; Jeffrey et al. 2009), or road traffic officials may even be underreporting TI to avoid criticism from superiors who expect to see rates go down, as one study suggested (Dandona et al. 2008).

The fact that males were substantially more likely to die from TI than females may be correlated to the fact that in some of these countries less women drive motorized vehicles; also in most cases women are accompanied by men outside of their houses.

Suboptimal public awareness of the importance of the issue has resulted in diminished emphasis on road safety policies at the national level in EMR countries. Lack of solid, reliable data may be a significant barrier to policymakers' prioritizing this major public health problem.

There are no definitive data on the number of people who survive with some form of permanent disability for every injury-related death, but estimates run between 10 and 50 times more permanent disabilities. As such, these injuries clearly contribute to the economic and social costs and have a negative impact on individuals, communities, and societies.

Many studies have shown that human behavioral factors collectively represent the main cause of three out of five road traffic crashes, and contribute to the cause in most remaining cases (Marshall et al. 1996; Evans 1996; Lyznicki et al. 1998; Sharma et al. 2002). A study in Saudi Arabia showed that more than $43 \%$ of unlicensed males drove a vehicle (El Bcheraoui et al. 2015). Among those male drivers (females are not allowed to drive by law), $86 \%$ engaged in at least one risky behavior while driving. Up to 95 and $98.5 \%$ of respondents reported not wearing a seat belt in the front (enforced by the law), and the back seat, respectively.

More attention must also be given to the needs of vulnerable road users, like pedestrians, children, and bicycle/motorcycle and public transport users. Making walking and cycling safer is critical to reducing the number of road traffic deaths and is important to promote non-motorized forms of transport.

\section{Conclusion}

Our study highlights the significant burden of TI deaths and injuries in the EMR countries, and the need for improving trauma centers and implementation of a faster emergency care in the EMR. Strict monitoring and enforcement of traffic laws, and programs to increase awareness and proper education for drivers should be developed jointly by the Ministries of Health, Interior Affairs, and Education and provided through their channels.

GBD 2015 Eastern Mediterranean Region Transportation Injuries Collaborators: Ali H. Mokdad, PhD (corresponding author), Institute for Health Metrics and Evaluation, University of Washington, Seattle, Washington, United States. Ibrahim Khalil, MD, Institute for Health Metrics and Evaluation, University of Washington, Seattle, Washington, United States. Charbel El Bcheraoui, PhD, Institute for Health Metrics and Evaluation, University of Washington, Seattle, Washington, United States. Raghid Charara, MD, American University of Beirut, Beirut, Lebanon. Maziar Moradi-Lakeh, MD, Department of Community Medicine, Preventive Medicine and Public Health Research Center, Gastrointestinal and Liver Disease Research Center (GILDRC), Iran University of Medical Sciences, Tehran, Iran. Ashkan Afshin, MD, Institute for Health Metrics and Evaluation, University of Washington, Seattle, Washington, United States. Nicholas J. Kassebaum, MD, Institute for Health Metrics and Evaluation, University of Washington, Seattle, Washington, United States; Department of Anesthesiology \& Pain Medicine, Seattle Children's Hospital, Seattle, Washington, United States. Michael Collison, BS, Institute for Health Metrics and Evaluation, University of Washington, Seattle, Washington, United States. Farah Daoud, BA/BS, Institute for Health Metrics and Evaluation, University of Washington. Adrienne Chew, ND, Institute for Health Metrics and Evaluation, University of Washington, Seattle, Washington, United States. Kristopher J. Krohn, BA, Institute for Health Metrics and Evaluation, University of Washington, Seattle, Washington, United States. Danny Colombara, $\mathrm{PhD}$, Institute for Health Metrics and Evaluation, University of Washington, Seattle, Washington, United States. Leslie Cornaby, BS, Institute for Health Metrics and Evaluation, University of Washington, Seattle, Washington, United States. Rebecca Ehrenkranz, MPH, Institute for Health Metrics and Evaluation, University of Washington, Seattle, Washington, United States. Nicholas Graetz, MPH, Institute for Health Metrics and Evaluation, University of Washington, Seattle, Washington, United States. Michael Kutz, BS, Institute for Health Metrics and Evaluation, University of Washington, Seattle, Washington, United States. Christopher Troeger, Institute for Health Metrics and Evaluation, University of Washington. Haidong Wang, $\mathrm{PhD}$, Institute for Health Metrics and Evaluation, University of Washington, Seattle, Washington, United States. Kalkidan Hassen Abate, MS, Jimma University, Jimma, Ethiopia. Foad Abd-Allah, MD, Department of Neurology, Cairo University, Cairo, Egypt. Abdishakur M. Abdulle, PhD, New York University, Abu Dhabi, United Arab Emirates. Semaw Ferede Abera, MSc, School of Public Health, College of Health Sciences, Mekelle University, Mekelle, Ethiopia; Food Security and Institute for Biological Chemistry and Nutrition, University of Hohenheim, Stuttgart, Germany. Aliasghar Ahmad Kiadaliri, PhD, Department of Clinical Sciences Lund, Orthopedics, Clinical Epidemiology Unit, Lund University, Lund, Sweden. Alireza Ahmadi, PhD, Kermanshah University of Medical Sciences, Kermanshah, Iran. Muktar Beshir Ahmed, MPH, College of Health Sciences, Department of Epidemiology, ICT and e-Learning Coordinator, Jimma University, Jimma, Ethiopia. Khurshid Alam, PhD, Murdoch Childrens Research 
Institute, The University of Melbourne, Parkville, Victoria, Australia; The University of Melbourne, Melbourne, VIC, Australia. The University of Sydney, Sydney, NSW, Australia. Deena Alasfoor, MSc, Ministry of Health, Al Khuwair, Muscat, Oman. Suliman Alghnam, PhD King Abdulah International Medical Research Center, Riyadh, Saudi Arabia; Center For Injury Research and Policy, Bloomberg School of Public Health, Johns Hopkins University, Baltimore, MD, United States. Raghib Ali, MSc, University of Oxford, Oxford, United Kingdom. Reza Alizadeh-Navaei, PhD, Gastrointestinal Cancer Research Center, Mazandaran University of Medical Sciences, Sari, Iran. Rajaa Al-Raddadi, PhD, Joint Program of Family and Community Medicine, Jeddah, Saudi Arabia. Ubai Alsharif, MPH, Charité Universitätsmedizin, Berlin, Germany. Khalid A. Altirkawi, MD, King Saud University, Riyadh, Saudi Arabia. Nahla Anber, PhD, Mansoura University, Mansoura, Egypt. Hossein Ansari, PhD, Health Promotion Research Center, Department of Epidemiology and Biostatistics, Zahedan University of Medical Sciences, Zahedan, Iran. Carl Abelardo T. Antonio, MD, Department of Health Policy and Administration, College of Public Health, University of the Philippines Manila, Manila, Philippines. Palwasha Anwari, MD Self-employed, Kabul, Afghanistan. Hamid Asayesh, $\mathrm{PhD}$, Department of Medical Emergency, School of Paramedic, Qom University of Medical Sciences, Qom, Iran. Tesfay Mehari Atey, MS, Mekelle University, Mekelle, Ethiopia. Leticia Avila-Burgos, PhD, National Institute of Public Health, Cuernavaca, Mexico. Suzanne L. Barker-Collo, PhD, School of Psychology, University of Auckland, Auckland, New Zealand. Shahrzad Bazargan-Hejazi, PhD, College of Medicine, Charles R. Drew University of Medicine and Science, Los Angeles, CA, United States; David Geffen School of Medicine, University of California at Los Angeles, Los Angeles, CA, United States. Neeraj Bedi, MD, College of Public Health and Tropical Medicine, Jazan, Saudi Arabia. Addisu Shunu Beyene, MPH, College of Health and Medical Science, Haramaya University, Harar, Ethiopia. Zulfiqar A. Bhutta, PhD, Centre of Excellence in Women and Child Health, Aga Khan University, Karachi, Pakistan; Centre for Global Child Health, The Hospital for Sick Children, Toronto, ON, Canada. Soufiane Boufous, PhD, Transport and Road Safety (TARS) Research, University of New South Wales, Sydney, New South Wales, Australia. Zahid A. Butt, PhD, Al Shifa Trust Eye Hospital, Rawalpindi, Pakistan. Carlos A. Castañeda-Orjuela, MSc, Colombian National Health Observatory, Instituto Nacional de Salud, Bogota, Colombia; Epidemiology and Public Health Evaluation Group, Public Health Department, Universidad Nacional de Colombia, Bogota, Colombia. Abdulaal A. Chitheer, MD, Ministry of Health, Baghdad, Iraq. Koustuv Dalal, $\mathrm{PhD}$, Centre for Injury Prevention and Safety Promotion, School of Health and Medical Sciences, Orebro University, Orebro, Sweden. Hadi Danawi, PhD, Walden University, Minneapolis, Minnesota, United States. Dragos V. Davitoiu, PhD, University of Medicine and Pharmacy Bucharest, Bucharest, Romania. Shirin Djalalinia, PhD, Undersecretary for Research \& Technology, Ministry of Health \& Medical Education, Tehran, Iran. Aman Yesuf Endries, MPH, Arba Minch University, Arba Minch, Ethiopia. Babak Eshrati, PhD, Ministry of Health and Medical Education, Tehran, Iran; Arak University of Medical Sciences, Arak, Iran. Alireza Esteghamati, MD, Endocrinology and Metabolism Research Center, Tehran University of Medical Sciences, Tehran, Iran. André Faro, PhD, Federal University of Sergipe, Aracaju, Brazil. Maryam S. Farvid, PhD, Department of Nutrition, Harvard T. H. Chan School of Public Health, Harvard University, Boston, MA, United States; Harvard/MGH Center on Genomics, Vulnerable Populations, and Health Disparities, Mongan Institute for Health Policy, Massachusetts General Hospital, Boston, MA, United States. Seyed-Mohammad Fereshtehnejad, PhD, Department of Neurobiology, Care Sciences and Society (NVS), Karolinska Institutet, Stockholm, Sweden. Florian Fischer, PhD, School of Public Health, Bielefeld University, Bielefeld, Germany. Wayne Gao, PhD, National Health Research
Institutes, Taipei, Taiwan. Solomon Weldemariam Gebrehiwot, MS, College of Health Sciences, Mekelle University, Mekelle, Ethiopia; Tsegaye Tewelde Gebrehiwot, MPH, Jimma University, Jimma, Ethiopia. Nima Hafezi-Nejad, MD, Endocrinology and Metabolism Research Center, Tehran University of Medical Sciences, Tehran, Iran. Hassan Haghparast Bidgoli, PhD, University College London, London, United Kingdom. Gessessew Bugssa Hailu, MSc Mekelle University, Mekelle, Ethiopia; Kilte Awlaelo Health and Demographic Surveillance System, Mekelle, Ethiopia. Randah Ribhi Hamadeh, DPhil, Arabian Gulf University, Manama, Bahrain. Samer Hamidi, DrPH, Hamdan Bin Mohammed Smart University, Dubai, United Arab Emirates. Delia Hendrie, MA, Centre for Population Health Research, Curtin University, Bentley, WA, Australia. Ileana Beatriz Heredia-Pi, PhD, National Institute of Public Health, Cuernavaca, Morelos, Mexico. Kathryn H. Jacobsen, PhD, Department of Global and Community Health, George Mason University, Fairfax, Virginia, United States. Spencer Lewis James, MD, Denver Health/ University of Colorado, Denver, CO, United States. Achala Upendra Jayatilleke, PhD, Postgraduate Institute of Medicine, Colombo, Sri Lanka; Institute of Violence and Injury Prevention, Colombo, Sri Lanka. Guohong Jiang, MD, Tianjin Centers for Disease Control and Prevention, Tianjin, China. Jost B. Jonas, MD, Department of Ophthalmology, Medical Faculty Mannheim, Ruprecht-Karls-University Heidelberg, Mannheim, Germany. Amir Kasaeian, PhD, HematologyOncology and Stem Cell Transplantation Research Center, Tehran University of Medical Sciences, Tehran, Iran; Endocrinology and Metabolism Population Sciences Institute, Tehran University of Medical Sciences, Tehran, Iran. Peter Njenga Keiyoro, PhD, Institute of Tropical and Infectious Diseases, Nairobi, Kenya; Kenya School of Continuing and Distance Education, Nairobi, Kenya. Yousef Saleh Khader, ScD, Department of Community Medicine, Public Health and Family Medicine, Jordan University of Science and Technology, Irbid, Jordan. Ejaz Ahmad Khan, MD, Health Services Academy, Islamabad, Punjab, Pakistan. Abdullah Tawfih Abdullah Khoja, MD, Mohammed Ibn Saudi University, Riyadh, Saudi Arabia. Ardeshir Khosravi, PhD, Iranian Ministry of Health and Medical Education, Tehran, Iran, Non-communicable Diseases Research Center, Tehran University of Medical Sciences, Tehran, Iran. Jagdish Khubchandani, $\mathrm{PhD}$, Department of Nutrition and Health Science, Ball State University, Muncie, Indiana, United States. Yun Jin Kim, PhD, Faculty of Chinese Medicine, Southern University College, Skudai, Johor, Malaysia. Soewarta Kosen, MD, Center for Community Empowerment, Health Policy and Humanities, National Institute of Health Research \& Development, Jakarta, Indonesia. Barthelemy Kuate Defo, PhD, Department of Social and Preventive Medicine, School of Public Health, University of Montreal, Montreal, Quebec, Canada; Department of Demography and Public Health Research Institute, University of Montreal, Montreal Canada. Heidi J. Larson, $\mathrm{PhD}$, Department of Infectious Disease Epidemiology, London School of Hygiene \& Tropical Medicine, London, United Kingdom; Institute for Health Metrics and Evaluation, University of Washington, Seattle, Washington, United States. Shai Linn, MD University of Haifa, Haifa, Israel. Raimundas Lunevicius, $\mathrm{PhD}$, Aintree University Hospital National Health Service Foundation Trust, Liverpool, United Kingdom; School of Medicine, University of Liverpool, Liverpool, United Kingdom. Hassan Magdy Abd El Razek, MBBCH, Mansoura Faculty of Medicine, Mansoura, Egypt. Mohammed Magdy Abd El Razek, MBBCH, Aswan University Hospital, Aswan Faculty of Medicine, Aswan, Egypt. Marek Majdan, PhD, Faculty of Health Sciences and Social Work, Department of Public Health, Trnava University, Trnava, Slovakia. Reza Majdzadeh, PhD, Knowledge Utilization Research Center and Community Based Participatory Research Center, Tehran University of Medical Sciences, Tehran, Iran. Azeem Majeed, MD, Department of Primary Care \& Public Health, Imperial College London, London, United Kingdom. Reza Malekzadeh, MD, Digestive Diseases Research Institute, Tehran 
University of Medical Sciences, Tehran, Iran. Peter Memiah, PhD, University of West Florida, Pensacola, FL, United States. Ziad A. Memish, MD, Saudi Ministry of Health, Riyadh, Saudi Arabia; College of Medicine, Alfaisal University, Riyadh, Saudi Arabia. Walter Mendoza, MD, United Nations Population Fund, Lima, Peru. Mubarek Abera Mengistie, MS, Jimma University, Jimma, Ethiopia. Tuomo J. Meretoja, PhD, Comprehensive Cancer Center, Breast Surgery Unit, Helsinki University Hospital, Helsinki, Finland; University of Helsinki, Helsinki, Finland. Ted R. Miller, PhD, Pacific Institute for Research \& Evaluation, Calverton, MD, United States; Centre for Population Health, Curtin University, Perth, WA, Australia. Shafiu Mohammed, PhD, Health Systems and Policy Research Unit, Ahmadu Bello University, Zaria, Nigeria; Institute of Public Health, Heidelberg University, Heidelberg, Germany. Ashagre Molla Assaye, MS, Bahir Dar University, Bahir Dar, Ethiopia. Carla Makhlouf Obermeyer, DSc, Center for Research on Population and Health, Faculty of Health Sciences, American University of Beirut, Beirut, Lebanon. Farshad Pourmalek, PhD, University of British Columbia, Vancouver, British Columbia, Canada. Mostafa Qorbani, $\mathrm{PhD}$, Non-communicable Diseases Research Center, Alborz University of Medical Sciences, Karaj, Iran. Amir Radfar, MD, A T Still University, Kirksville, MO, United States. Anwar Rafay, MS, Contech International Health Consultants, Lahore, Pakistan; Contech School of Public Health, Lahore, Pakistan. Vafa Rahimi-Movaghar, MD, Sina Trauma and Surgery Research Center, Tehran University of Medical Sciences, Tehran, Iran. Mahfuzar Rahman, PhD, Research and Evaluation Division, BRAC, Dhaka, Bangladesh. Rajesh Kumar Rai, MPH, Society for Health and Demographic Surveillance, Suri, India. Kavitha Ranganathan, MD, University of Michigan Health Systems, Ann Arbor, Michigan, United States. David Laith Rawaf, MD, WHO Collaborating Centre, Imperial College London, London, United Kingdom, North Hampshire Hospitals, Basingstroke, United Kingdom; University College London Hospitals, London, United Kingdom. Salman Rawaf, MD, Imperial College London, London, United Kingdom. Amany H. Refaat, PhD, Walden University, Minneapolis, MN, United States; Suez Canal University, Ismailia, Egypt. Andre M. N. Renzaho, PhD, Western Sydney University, Penrith, NSW, Australia. Satar Rezaei, PhD, School of Public Health, Kermanshah University of Medical Sciences, Kermanshah, Iran. David Rojas-Rueda, PhD, Campus MAR, Barcelona Biomedical Research Park (PRBB), ISGlobal Instituto de Salud Global de Barcelona, Catalonia, Spain. Gholamreza Roshandel, PhD, Golestan Research Center of Gastroenterology and Hepatology, Golestan University of Medical Sciences, Gorgan, Iran; Digestive Diseases Research Institute, Tehran University of Medical Sciences, Tehran, Iran. Mahdi Safdarian, MD, Sina Trauma \& Surgery Research Center, Tehran University of Medical Sciences, Tehran, Iran. Saeid Safiri, PhD, Managerial Epidemiology Research Center, Department of Public Health, School of Nursing and Midwifery, Maragheh University of Medical Sciences, Maragheh, Iran. Mohammad Ali Sahraian, MD, MS Research Center, Neuroscience Institute, Tehran University of Medical Sciences, Tehran, Iran. Payman Salamati, MD, Sina Trauma and Surgery Research Center, Tehran University of Medical Sciences, Tehran, Iran. Abdallah M. Samy, PhD, Ain Shams University, Cairo, Egypt. Juan Ramon Sanabria, MD, J Edwards School of Medicine, Marshall Univeristy, Huntington, WV, United States; Case Western Reserve University, Cleveland, OH, United States. Milena M. Santric Milicevic, PhD, Institute of Social Medicine, Faculty of Medicine, University of Belgrade, Belgrade, Serbia, Centre School of Public Health and Health Management, Faculty of Medicine, University of Belgrade, Belgrade, Serbia. Benn Sartorius, PhD, Public Health Medicine, School of Nursing and Public Health, University of KwaZulu-Natal, Durban, South Africa; UKZN Gastrointestinal Cancer Research Centre, South African Medical Research Council (SAMRC), Durban, South Africa. David C. Schwebel, PhD, University of Alabama at Birmingham, Birmingham, Alabama,
United States. Sadaf G. Sepanlou, PhD, Digestive Diseases Research Institute, Tehran University of Medical Sciences, Tehran, Iran. Amira Shaheen, PhD, Department of Public Health, An-Najah University, Nablus, Palestine. Masood Ali Shaikh, MD, Independent Consultant, Karachi, Pakistan. Mansour Shamsipour, PhD, Institute for Environmental Research, Tehran University of Medical Sciences, Tehran, Iran. Morteza Shamsizadeh, MPH, Department of Medical Surgical Nursing, School of Nursing and Midwifery, Hamadan University of Medical Sciences, Hamadan, Iran. Badr H. A. Sobaih, MD, King Saud University, Riyadh, Saudi Arabia. Muawiyyah Babale Sufiyan, MBA, Ahmadu Bello University, Zaria, Nigeria. Jacob E. Sunshine, MD, University of Washington, Seattle, Washington, United States. Arash Tehrani-Banihashemi, PhD, Preventive Medicine and Public Health Research Center, Iran University of Medical Sciences, Tehran, Iran; Community Medicine Department, Iran University of Medical Sciences, Tehran, Iran. Mohamad-Hani Temsah, MD, King Saud University, Riyadh, Saudi Arabia. Abdullah Sulieman Terkawi, MD, Department of Anesthesiology, University of Virginia, Charlottesville, VA, United States; Department of Anesthesiology, King Fahad Medical City, Riyadh, Saudi Arabia; Outcomes Research Consortium, Cleveland Clinic, Cleveland, OH, United States. J. S. Thakur, MD, School of Public Health, Post Graduate Institute of Medical Education and Research, Chandigarh, Union Territory Chandigarh, India. Roman Topor-Madry, PhD, Institute of Public Health, Faculty of Health Sciences, Jagiellonian University Medical College, Kraków, Poland; Faculty of Health Sciences, Wroclaw Medical University, Wroclaw, Poland. Olalekan A. Uthman, PhD, Warwick Medical School, University of Warwick, Coventry, United Kingdom. Vasiliy Victorovich Vlassov, MD, National Research University Higher School of Economics, Moscow, Russia. Stein Emil Vollset, DrPH, Center for Disease Burden, Norwegian Institute of Public Health, Bergen, Norway; Department of Global Public Health and Primary Care, University of Bergen, Bergen, Norway; Institute for Health Metrics and Evaluation, University of Washington, Seattle, Washington, United States. Tolassa Wakayo, MS, Jimma University, Jimma, Ethiopia. Andrea Werdecker, PhD, Competence Center Mortality-Follow-Up of the German National Cohort, Federal Institute for Population Research, Wiesbaden, Germany. Mohsen Yaghoubi, MSc, School of Public Health, University of Saskatchewan, Saskatoon, Saskatchewan, Canada. Mehdi Yaseri, PhD, Tehran University of Medical Sciences, Tehran, Iran; Ophthalmic Research Center, Shahid Beheshti University of Medical Sciences, Tehran, Iran. Naohiro Yonemoto, MPH, Department of Biostatistics, School of Public Health, Kyoto University, Kyoto, Japan. Mustafa Z. Younis, DrPH, Jackson State University, Jackson, MS, United States. Maysaa El Sayed Zaki, PhD, Faculty of Medicine, Mansoura University, Mansoura, Egypt. Aisha O. Jumaan, PhD, Independent Consultant, Seattle, Washington, United States. Theo Vos, PhD, Institute for Health Metrics and Evaluation, University of Washington, Seattle, Washington, United States. Simon I. Hay, DSc, Oxford Big Data Institute, Li Ka Shing Centre for Health Information and Discovery, University of Oxford, Oxford, United Kingdom, Institute for Health Metrics and Evaluation, University of Washington, Seattle, Washington, United States. Mohsen Naghavi, PhD, Institute for Health Metrics and Evaluation, University of Washington, Seattle, Washington, United States. Christopher J. L. Murray, DPhil, Institute for Health Metrics and Evaluation, University of Washington, Seattle, Washington, United States.

\section{Compliance with ethical standards}

This manuscript reflects original work that has not previously been published in whole or in part and is not under consideration elsewhere. All authors have read the manuscript and have agreed that the work is ready for submission and accept responsibility for its contents. 
Ethical approval The authors of this paper have complied with all ethical standards and do not have any conflicts of interest to disclose at the time of submission. The funding source played no role in the design of the study, the analysis and interpretation of data, and the writing of the paper. The study did not involve human participants and/or animals; therefore, no informed consent was needed.

Funding This research was funded by the Bill \& Melinda Gates Foundation.

Conflict of interest The authors declare that they have no conflicts of interest at this time.

Open Access This article is distributed under the terms of the Creative Commons Attribution 4.0 International License (http://crea tivecommons.org/licenses/by/4.0/), which permits unrestricted use, distribution, and reproduction in any medium, provided you give appropriate credit to the original author(s) and the source, provide a link to the Creative Commons license, and indicate if changes were made.

\section{References}

Ainy E, Soori H, Ganjali M et al (2014) Estimating cost of road traffic injuries in Iran using willingness to pay (WTP) method. PLoS One 9:e112721. doi:10.1371/journal.pone.0112721

Ameratunga S, Hijar M, Norton R (2006) Road-traffic injuries: confronting disparities to address a global-health problem. The Lancet 367:1533-1540. doi:10.1016/S0140-6736(06)68654-6

Barrimah I, Midhet F, Sharaf F (2012) Epidemiology of road traffic injuries in Qassim region, Saudi Arabia: consistency of police and health data. Int J Health Sci 6:31-41

Bishai D, Quresh A, James P, Ghaffar A (2006) National road casualties and economic development. Health Econ 15:65-81. doi: $10.1002 /$ hec. 1020

Byass P, de Courten M, Graham WJ et al (2013) Reflections on the global burden of disease 2010 estimates. PLoS Med 10:e1001477. doi:10.1371/journal.pmed.1001477

Chandran A, Hyder AA, Peek-Asa C (2010) The global burden of unintentional injuries and an agenda for progress. Epidemiol Rev 32:110-120. doi:10.1093/epirev/mxq009

Dandona R, Kumar GA, Ameer MA et al (2008) Underreporting of road traffic injuries to the police: results from two data sources in urban India. Inj Prev J Int Soc Child Adolesc Inj Prev 14:360-365. doi:10.1136/ip.2008.019638

El Bcheraoui C, Basulaiman M, Tuffaha M et al (2015) Get a license, buckle up, and slow down: risky driving patterns among Saudis. Traffic Inj Prev 16:587-592. doi:10.1080/15389588.2014. 990090

Evans L (1996) The dominant role of driver behavior in traffic safety. Am J Public Health 86:784-786

GBD 2015 Disease and Injury Incidence and Prevalence Collaborators (2016) Global, regional, and national incidence, prevalence, and years lived with disability for 310 diseases and injuries, 1990-2015: a systematic analysis for the Global Burden of Disease Study 2015. Lancet Lond Engl 388:1545-1602. doi:10. 1016/S0140-6736(16)31678-6

Hyder AA, Vecino-Ortiz AI (2014) BRICS: opportunities to improve road safety. Bull World Health Organ 92:423-428. doi:10.2471/ BLT.13.132613

Jeffrey S, Stone DH, Blamey A et al (2009) An evaluation of police reporting of road casualties. Inj Prev J Int Soc Child Adolesc Inj Prev 15:13-18. doi:10.1136/ip.2008.018630
Joubert J, Rao C, Bradshaw D et al (2012) Characteristics, availability and uses of vital registration and other mortality data sources in post-democracy South Africa. Glob Health Action. doi:10.3402/ gha.v5i0.19263

Karacasu M, Er A, Bilgiç S, Barut HB (2011) Variations in traffic accidents on seasonal, monthly, daily and hourly basis: Eskisehir case. Proc Soc Behav Sci 20:767-775. doi:10.1016/j.sbspro. 2011.08.085

Kassebaum NJ, Arora M, Barber RM et al (2016) Global, regional, and national disability-adjusted life-years (DALYs) for 315 diseases and injuries and healthy life expectancy (HALE), 1990-2015: a systematic analysis for the Global Burden of Disease Study 2015. The Lancet 388:1603-1658. doi:10.1016/ S0140-6736(16)31460-X

Keay K, Simmonds I (2005) The association of rainfall and other weather variables with road traffic volume in Melbourne, Australia. Accid Anal Prev 37:109-124. doi:10.1016/j.aap. 2004.07.005

Loo BPY, Tsui KL (2007) Factors affecting the likelihood of reporting road crashes resulting in medical treatment to the police. Inj Prev 13:186-189. doi:10.1136/ip.2006.013458

Luoma J, Sivak M (2012) Road-safety management in Brazil, Russia, India, and China https://deepblue.lib.umich.edu/bitstream/han dle/2027.42/89427/102786.pdf?sequence=1\&isAllowed=y. Accessed 13 Jul 2017

Lyznicki JM, Doege TC, Davis RM, Williams MA (1998) Sleepiness, driving, and motor vehicle crashes. Council on scientific affairs, American Medical Association. JAMA 279:1908-1913

Mahapatra P, Shibuya K, Lopez AD et al (2007) Civil registration systems and vital statistics: successes and missed opportunities. The Lancet 370:1653-1663. doi:10.1016/S0140-6736(07)613087

Marshall C, Boyd KT, Moran CG (1996) Injuries related to car crime: the joy-riding epidemic. Injury 27:79-80

Mathers CD, Salomon JA, Ezzati M et al (2006) Sensitivity and uncertainty analyses for burden of disease and risk factor estimates. In: Global Burden of Disease and Risk Factors. Oxford University Press, New York, pp399-426

Mock CN, Adzotor KE, Conklin E et al (1993) Trauma outcomes in the rural developing world: comparison with an urban level I trauma center. J Trauma Acute Care Surg 35:518-523

Mokdad AH, Forouzanfar MH, Daoud F, et al (2016) Health in times of uncertainty in the eastern Mediterranean region, 1990-2013: a systematic analysis for the Global Burden of Disease Study 2013. Lancet GlobHealth 4:e704-e713. doi:10.1016/S2214109X(16)30168-1

Mokdad AH, Jaber S, Aziz MIA, et al (2014) The state of health in the Arab world, 1990-2010: an analysis of the burden of diseases, injuries, and risk factors. The Lancet 383:309-320. doi:10.1016/S0140-6736(13)62189-3

Naeem Z (2010) Road traffic injuries - changing trend? Int J Health Sci 4(2):v-viii

Naghavi M, Shahraz S, Bhalla K et al (2009) Adverse health outcomes of road traffic injuries in Iran after rapid motorization. Arch Iran Med 12:284-294

Noland RB (2003) Medical treatment and traffic fatality reductions in industrialized countries. Accid Anal Prev 35:877-883

Obermeyer Z, Rajaratnam JK, Park CH et al (2010) Measuring adult mortality using sibling survival: a new analytical method and new results for 44 Countries, 1974-2006. PLOS Med 7:e1000260. doi:10.1371/journal.pmed.1000260

Razzak JA, Luby SP, Laflamme L, Chotani H (2004) Injuries among children in Karachi, Pakistan - what, where and how. Public Health 118:114-120. doi:10.1016/S0033-3506(03)00147-1

Reynolds TA, Stewart B, Drewett I et al (2017) The impact of trauma care systems in low- and middle-income Countries. Annu Rev 
Public Health 38:507-532. doi:10.1146/annurev-publhealth032315-021412

Setel PW, Macfarlane SB, Szreter S et al (2007) A scandal of invisibility: making everyone count by counting everyone. The Lancet 370:1569-1577. doi:10.1016/S0140-6736(07)61307-5

Sharma B, Gautam C, Singh R (2002) Road traffic accidents vis-à-vis problems related to ageing. Hosp Today 7:523-528

Sukhai A, Jones AP, Love BS, Haynes R (2011) Temporal variations in road traffic fatalities in South Africa. Accid Anal Prev 43:421-428. doi:10.1016/j.aap.2010.09.012

Wang H, Naghavi M, Allen C et al (2016) Global, regional, and national life expectancy, all-cause mortality, and cause-specific mortality for 249 causes of death, 1980-2015: a systematic analysis for the Global Burden of Disease Study 2015. The Lancet 388:1459-1544. doi:10.1016/S0140-6736(16)31012-1

WHO (2013) Global status report on road safety: supporting a decade of action. http://www.who.int/violence_injury_prevention/road_ safety_status/2013/en/. Accessed 13 Jul 2017

WHO (2015) Global status report on road safety 2015. http://www. who.int/violence_injury_prevention/road_safety_status/2015/en/ . Accessed 13 Jul 2017

WHO Regional Office for the Eastern Mediterranean (2017) Home. http://www.emro.who.int/index.html. Accessed 13 Jul 2017 